\title{
Comparative Evaluation of the Effect of Two Desensitizing Dentifrices containing Novamin Technology and Pro-Argin Technology following Scaling and Root Planing
}

\author{
${ }^{1}$ Pooja R Vazhakkat, ${ }^{2}$ KS Shobha
}

\begin{abstract}
Aim: To compare the desensitizing efficacy of two commercially available dentifrices, one containing Novamin technology (calcium sodium phosphosilicate, a bioactive glass) and the other containing Pro-Argin technology (arginine and calcium carbonate) when applied after scaling and root planing.

Materials and methods: About 30 subjects having at least two sensitive teeth (total 60 sites) after scaling and root planing were included in this study and randomly divided into two groups, each containing 15 patients: Group I received Pro-Argin technology (arginine and calcium carbonate) and group II received Novamin technology (calcium sodium phosphosilicate, a bioactive glass) containing desensitizing toothpaste. The sensitive teeth were selected on the basis of Schiff cold air sensitivity scale (SCASS) with an air blast hypersensitivity score of 2 or 3 and cold water test with visual analog scale (VAS) score of 4 to 10 responses. The subjects' response was recorded at baseline (i.e., immediately after treatment) and after 1, 2, and 4 weeks respectively, using the SCASS and VAS scale.
\end{abstract}

Results: Using Mann-Whitney test, we found no statistically significant differences in the score between the two desensitizing toothpastes. But, there was a statistically significant difference between the baseline and follow-up scores done consecutively for individual toothpastes.

Conclusion: Therefore, within the limitations of the study, we found no statistically significant difference in the efficacy of the two desensitizing toothpastes.

Keywords: Dentifrices, Novamin technology, Visual analog scale.

How to cite this article: Vazhakkat PR, Shobha KS. Comparative Evaluation of the Effect of Two Desensitizing Dentifrices containing Novamin Technology and Pro-Argin Technology following Scaling and Root Planing. J Health Sci Res 2017;8(1):7-14.

\section{Source of support: Nil}

Conflict of interest: None

\section{INTRODUCTION}

Dentin hypersensitivity $(\mathrm{DH})$ is a significant clinical oral problem, affecting many adults worldwide. It is defined as

\footnotetext{
${ }^{1}$ Postgraduate Student, ${ }^{2}$ Reader

1,2Department of Periodontics, The Oxford Dental College \& Hospital, Bengaluru, Karnataka, India

Corresponding Author: Pooja R Vazhakkat, Postgraduate Student, Department of Periodontics, The Oxford Dental College \& Hospital, Bengaluru, Karnataka, India, Phone: +91-9880403091, e-mail: vazhakkatpooja@gmail.com
}

a brief, sharp, well-localized pain in response to thermal, evaporative, tactile, osmotic, or chemical stimuli, which cannot be referred to any other form of dental defect or pathology. ${ }^{1,2}$ Dentin hypersensitivity is a symptom complex, rather than disease and a persisting problem, which without proper clinical management can have a significant impact on a sufferer's quality of life. ${ }^{3,4}$

The prevalence of dentinal hypersensitivity has been reported over the years and is between 8 and $57 \%$ of the adult dentate population and up to $30 \%$ of adults suffer at some point in their lifetime. Dentinal hypersensitivity has been shown to peak in 20 to 30 years old and then rise again when in their $50 \mathrm{~s} .{ }^{5}$

Etiological factors relevant to the development of DH include erosive wear close to the gingival margin where the enamel is thinnest, its removal resulting in exposure of the underlying dentin. ${ }^{4}$ Gingival recession resulting from periodontal disease or toothbrushing trauma has also been considered to be an etiological factor in $\mathrm{DH}$, as this may result in the exposure of the tooth root and associated cementum. Relative to enamel, the cementum is more susceptible to removal through erosive wear, a process that rapidly leads to exposure of the underlying dentin. ${ }^{6,7}$ Periodontal procedures like scaling and root planing and periodontal surgery are also known to increase sensitivity in a transient manner. ${ }^{8,9}$

Various mechanisms have been proposed to explain the development of DH. Most accepted of these is the hydrodynamic theory which was first explained by Gysi in 1900 and the experimental evidence for which was provided by Bränström. According to this theory, the movement of dentinal fluid on stimulation with thermal, chemical, evaporative, or electric stimulus is responsible for excitation of the underlying dentinal mechanoreceptor resulting in sensitivity. Occlusion of dentinal tubule by various occluding agents, application of anti-inflammatory agents, as well as root covering by periodontal surgery are treatment approaches to reduce $\mathrm{DH}^{10}$

A novel DH treatment technology (Pro-Argin), consisting of $8 \%$ arginine and calcium carbonate, mimics the natural process of plugging patent dentin tubules. When applied to exposed dentin, the open dentin tubules are sealed with a plug that contains arginine, calcium, phosphate, and carbonate. The essential components of 
this new technology are arginine, an amino acid which is positively charged at physiological $\mathrm{pH}$, i.e., $\mathrm{pH} 6.5$ to 7.5 , bicarbonate, a $\mathrm{pH}$ buffer, and calcium carbonate, a source of calcium. This technology, called Pro-Argin, has been shown to physically plug and seal exposed dentin tubules and to effectively relieve $\mathrm{DH}{ }^{11,12}$

Calcium sodium phosphosilicate (Novamin) is one of the latest advances that was developed for use in oral health care, in reducing dentinal hypersensitivity. It physically occludes the dentinal tubules. Novamin is a bioactive glass in the class of highly biocompatible materials that were originally developed as bone regenerative materials. These materials are reactive when exposed to body fluids and deposit hydroxycarbonate apatite, a mineral, i.e., chemically similar to the mineral in enamel and dentin. ${ }^{13,14}$ When incorporated into a dentifrice, particles are deposited onto the dentin surface to mechanically occlude the dentinal tubules. The physical occlusion of Novamin particles begins when the material is subjected to an aqueous environment; sodium ions in the particles immediately begin to exchange with hydrogen cations. This rapid release of ions allows calcium ions in the particle structure, as well as phosphate ions to be released from the material. This initial series of reactions occurs within seconds of exposure and the release of calcium and phosphorous ions continues as long as the particles are exposed to the aqueous environment. ${ }^{14}$ A localized and transient increase in $\mathrm{pH}$ occurs during the initial exposure of the material due to the release of sodium. The increase in $\mathrm{pH}$ helps to precipitate the calcium and phosphate ions from the Novamin particle, along with calcium and phosphorous found in saliva, to form a calcium phosphate layer. As the deposition of calcium and phosphorous complexes continues, this layer crystallizes into hydroxycarbonate apatite, which is chemically and structurally equivalent to the biological apatite. The combination of the residual Novamin particles and the hydroxyapatite layer results in the physical occlusion of dentinal tubules, which will relieve hypersensitivity. ${ }^{15}$

The present study aims to compare the desensitizing efficacy of two commercially available dentifrices, one containing Novamin technology (calcium sodium phosphosilicate, a bioactive glass) and the other containing Pro-Argin technology (arginine and calcium carbonate) when applied after scaling and root planing.

\section{MATERIALS AND METHODS}

The study protocol was approved by the ethical committee of The Oxford Dental College and Hospital, Bengaluru. About 40 patients who visited the Department of Periodontics, The Oxford Dental College and Hospital, Bengaluru, after satisfying the inclusion and exclusion criteria were recruited in the study. All the patients were given a detailed verbal and written description of the study, and they signed a written informed consent form prior to commencement of the study. These patients were divided into two groups. About 30 subjects having at least two sensitive teeth after scaling and root planing were randomly divided into two groups each containing 15 patients:

Group I received Pro-Argin technology ( $8 \%$ arginine and calcium carbonate)

Group II received Novamin technology (calcium sodium phosphosilicate, a bioactive glass) containing desensitizing toothpaste

\section{Inclusion Criteria}

- Aged between 18 and 65 years.

- Complaining of sensitivity on at least two teeth after scaling and root planing with a hypersensitivity score of 2 or 3 by Schiff cold air sensitivity scale (SCASS).

\section{Exclusion Criteria}

- Dental pathology causing pain similar to $\mathrm{DH}$.

- Active cervical caries, deep abrasion requiring class V filling, chipped tooth, erosion, or abfraction, recession.

- Used/using any type of desensitizing agent for last 6 months.

- On anti-inflammatory and analgesic medications.

- Pregnant or lactating females.

- Undergone any periodontal surgery in last 6 months.

- Undergoing orthodontic therapy.

- Patients with history of gastroesophageal reflux disorder

- Allergy to contents of toothpaste.

Patients underwent scaling and root planing. Preoperative and postoperative photographs were taken (Figs 1 to 6 ). The sensitive teeth were selected on the basis of schiff cold air sensitivity scale with an air blast hypersensitivity score of 2 or 3 . The subjects response was recorded at baseline (i.e., immediately after treatment) (Fig. 7), after 1 (Fig. 8), 2 (Fig. 9), and 4 (Fig. 10) weeks respectively using schiff cold air sensitivity scale.

\section{Air Blast Stimulation}

Adjacent teeth were isolated by placement of the examiners gloved fingers over the teeth. Air was delivered from a standardized dental unit air syringe at 38 psi. The air was directed at the exposed surface of hypersensitive tooth for 1 second from a distance of approximately $1 \mathrm{~cm}$.

\section{Schiff Cold Air Sensitivity Scale ${ }^{16}$}

- $0=$ Tooth/subject does not respond to air stimulus.

- $1=$ Tooth/subject responds to air stimulus but does not request discontinuation of stimulus. 


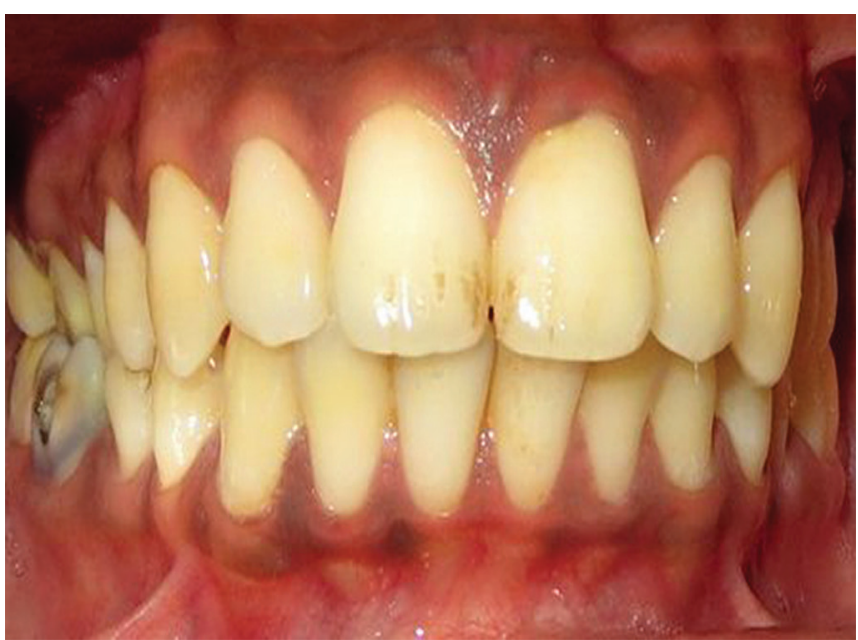

Fig. 1: Preoperative frontal view

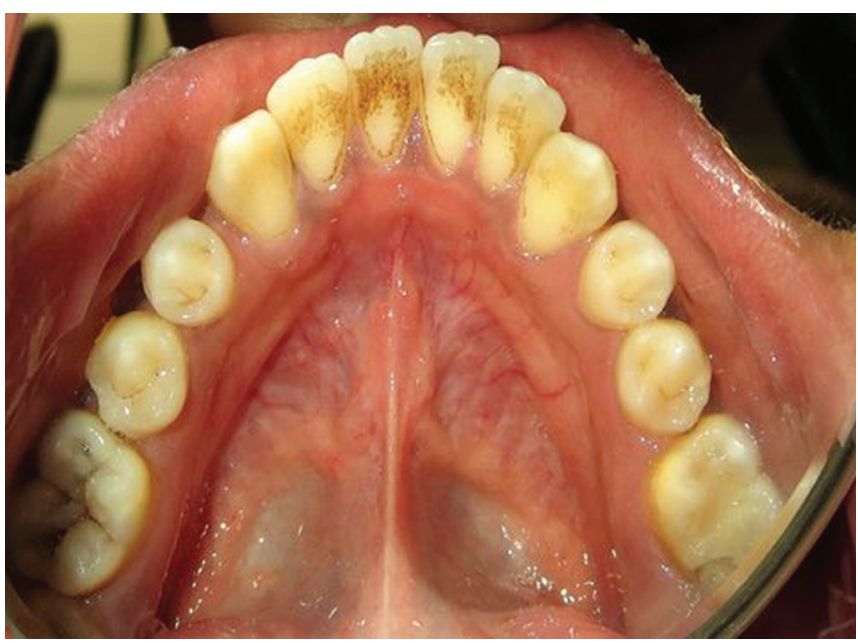

Fig. 3: Preoperative lingual view

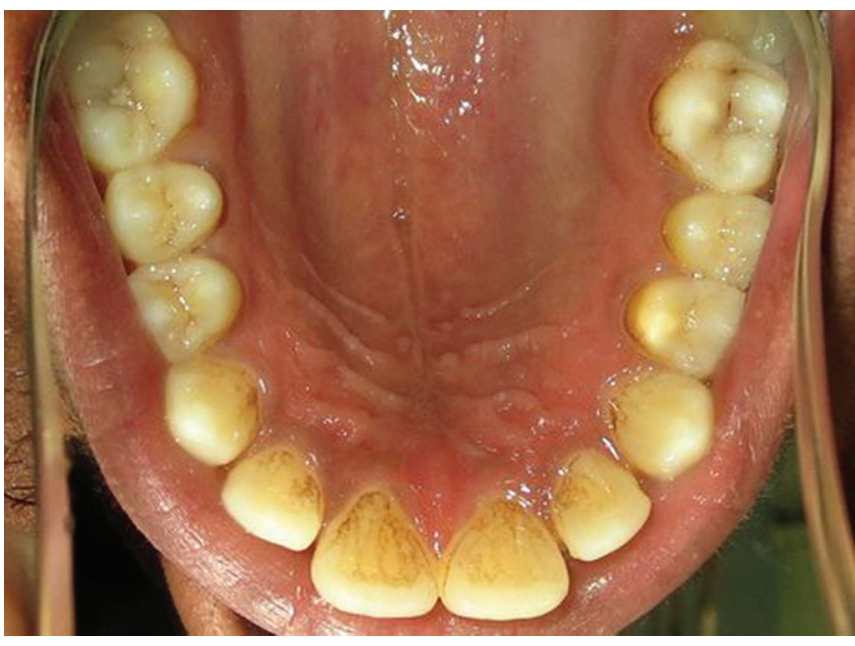

Fig. 5: Preoperative palatal view

$2=$ Tooth/subject responds to air stimulus and requests discontinuation or moves from stimulus.

$3=$ Tooth/subject responds to air stimulus, considers stimulus to be painful, and requests discontinuation of stimulus.

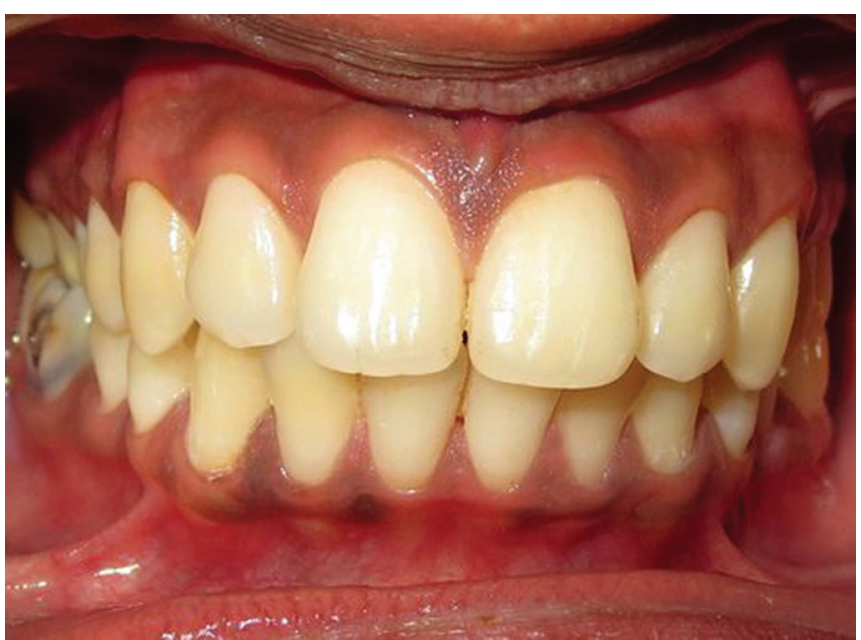

Fig. 2: Postoperative frontal view

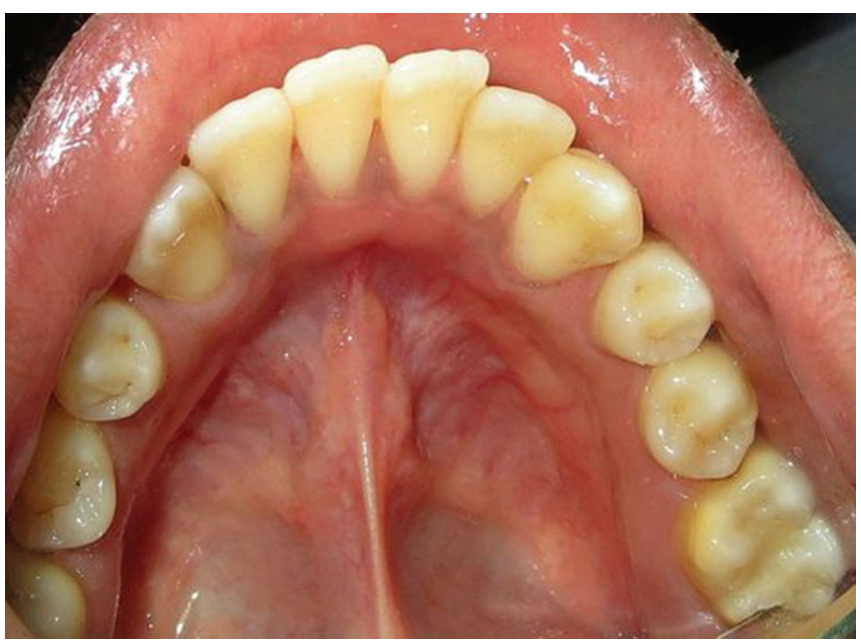

Fig. 4: Postoperative lingual view

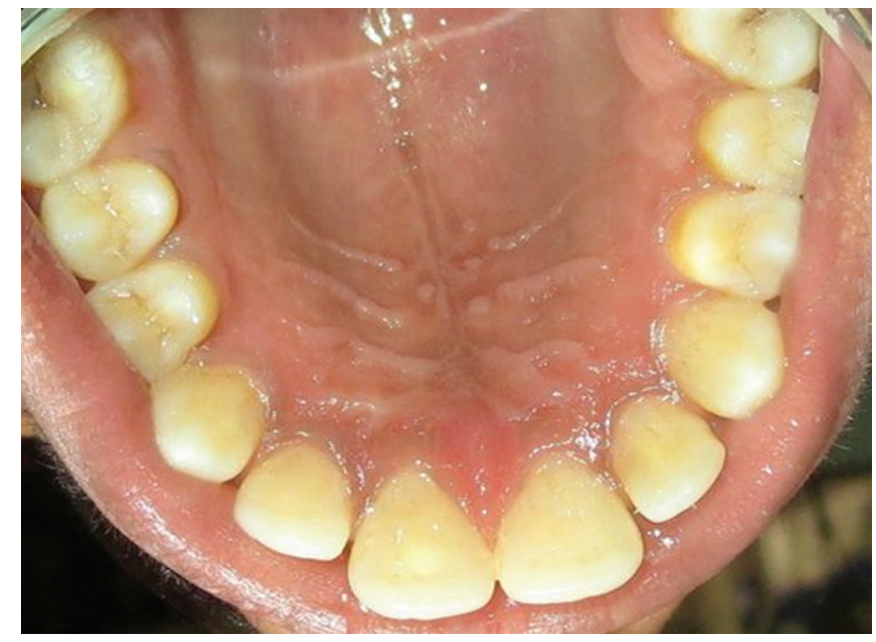

Fig. 6: Postoperative palatal view

\section{Cold Water Test}

The cold water test was performed approximately 10 minutes after the air blast test. Cold water was delivered as $1 \mathrm{~mL}$ of freshly melted ice cold water immediately 


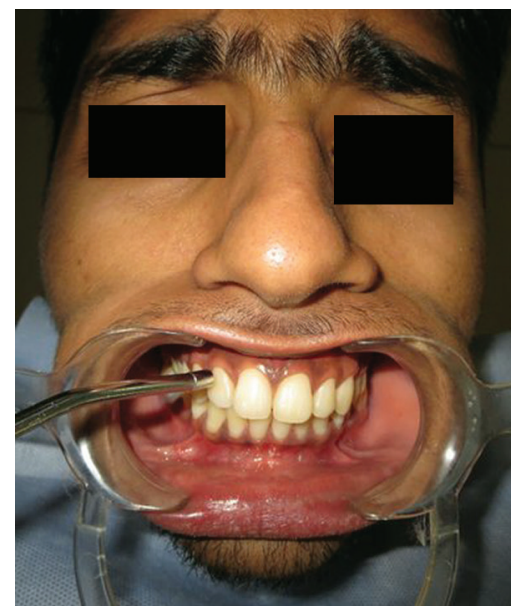

Fig. 7: Schiff cold air sensitivity test (baseline)

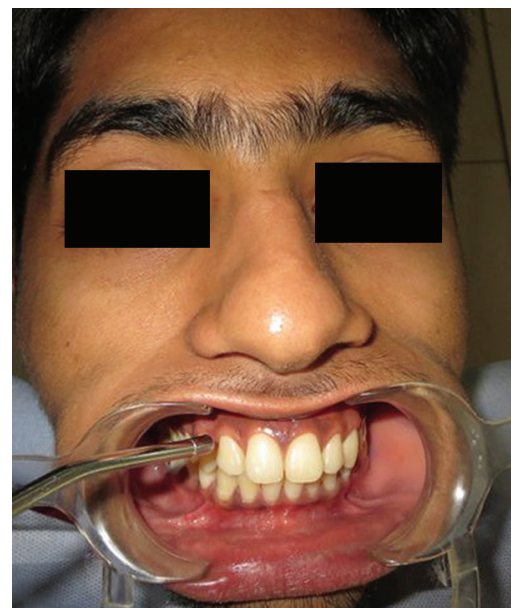

Fig. 9: Schiff cold air sensitivity test (2nd week)

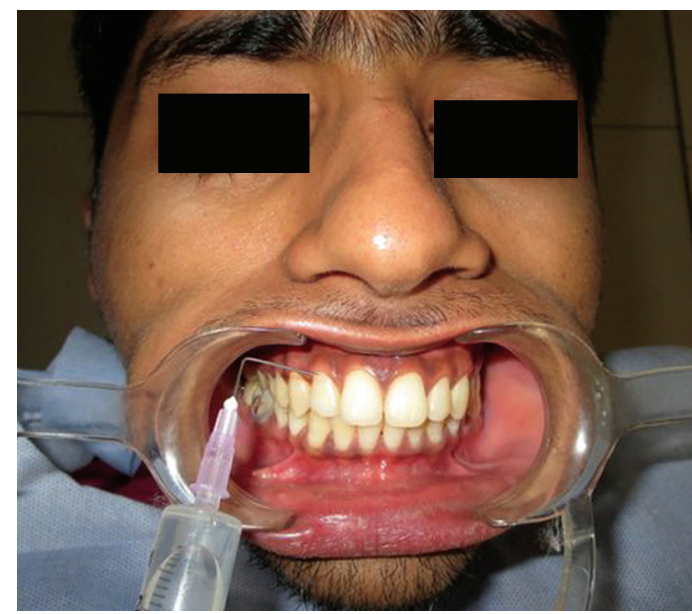

Fig. 11: Cold water test baseline

on the sensitive tooth using a syringe. Patient's response on visual analog scale (VAS) of $10 \mathrm{~cm}$ was measured.

The subjects response was recorded at baseline (i.e., immediately after treatment) (Fig. 11), after 1 (Fig. 12), 2 (Fig. 13), and 4 (Fig. 14) weeks, respectively.

The test was repeated three times before a score using VAS was noted. VAS score of 4 to 10 responses were selected (0—no pain, 10 — severe pain) ${ }^{17}$

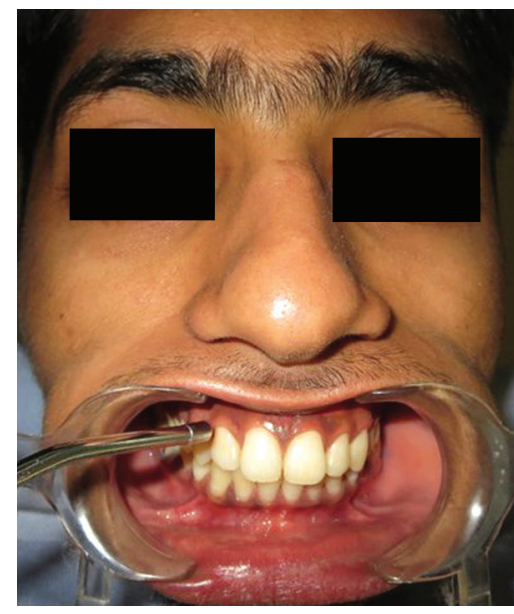

Fig. 8: Schiff cold air sensitivity test (1st week)

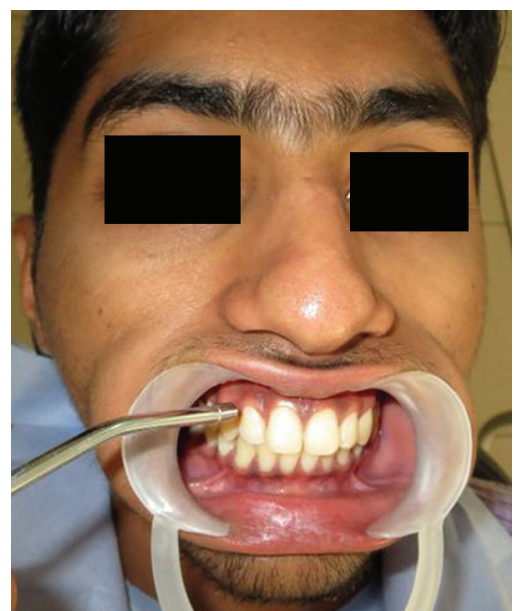

Fig. 10: Schiff cold air sensitivity test (4th week)

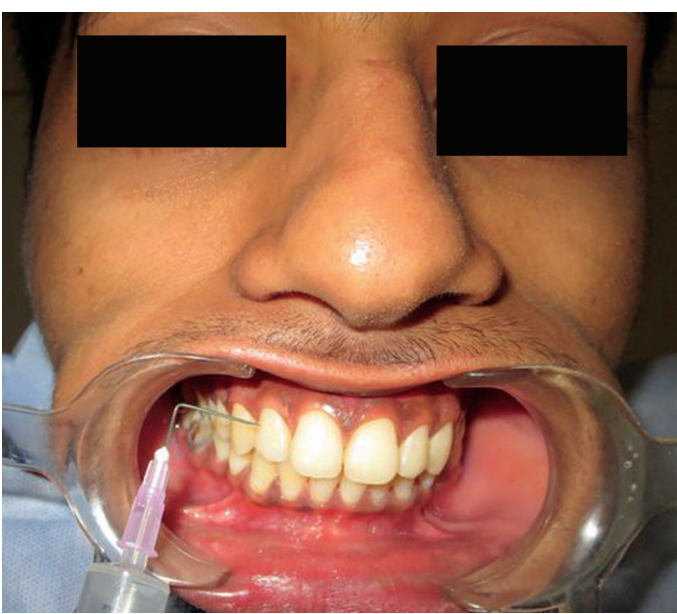

Fig. 12: Cold water test (1st week)

Patients were provided with the respective dentifrice. They were instructed to brush for 1 minute in their usual manner, twice daily throughout the period of their study, and asked to refrain from consuming very hot, cold, sweet, or sour food or drinks. Subjects were also directed to refrain from any other dentifrice or mouthrinse during the trial but were allowed to continue their normal oral hygiene practice. Assessment was performed again at 2 and 4 weeks. 


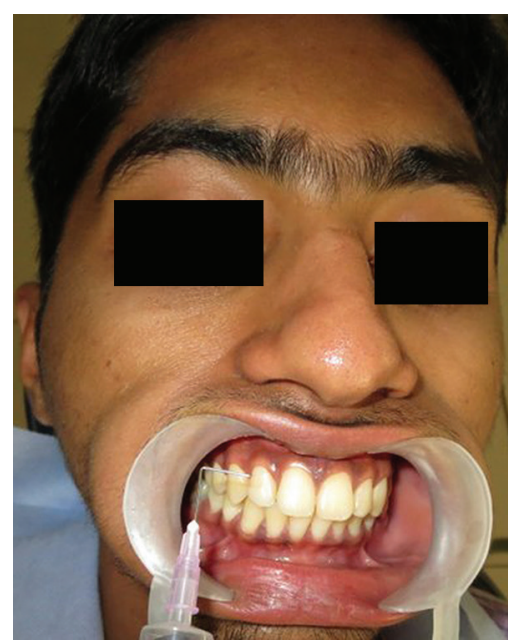

Fig. 13: Cold water test (2nd week)

\section{Statistical Analysis}

The main focus of the statistical analysis was the difference in change rates (VAS and SCASS) between the two groups. Intergroup comparison was done using Wilcoxon signed rank test.

- Null hypothesis: There is no significant difference in the score between the two technologies, i.e., $\eta_{1}=\eta_{2}$

- Alternate hypothesis: There is a significant difference in the score recorded between the two technologies, i.e., $\eta_{1} \neq \eta_{2}$

- Level of significance: $\mathrm{p}=0.05$

- Statistical test used: Mann-Whitney test

- Decision criteria were made by comparing the $p$ value with the level of significance. If $p<0.05$, we reject the null hypothesis and accept the alternate hypothesis. If $\mathrm{p} \geq 0.05$, we accept the null hypothesis.

\section{RESULTS}

The purpose of this study was to compare the desensitizing efficacy of two commercially available dentifrices, one containing Novamin technology (calcium sodium phosphosilicate, a bioactive glass) and the other containing

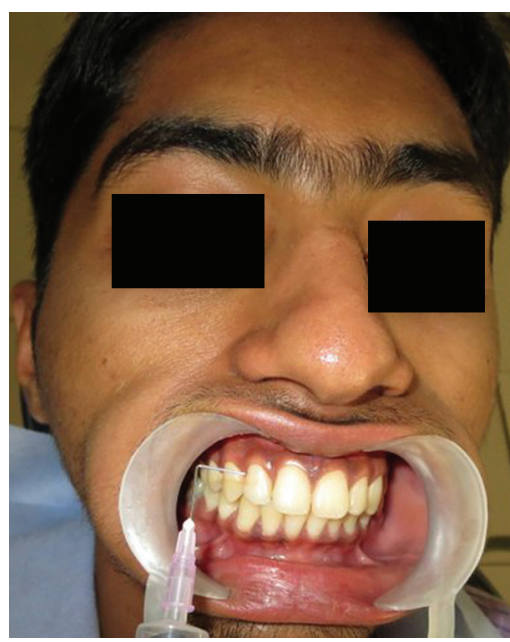

Fig. 14: Cold water test (4th week)

Pro-Argin technology (arginine and calcium carbonate) when applied after scaling and root planing.

A total of 30 patients (19 males and 11 females) in the age range of 18 to 60 years were selected on basis of the inclusion and exclusion criteria.

An informed written consent was obtained from each patient after explaining the study design. Patients having at least two sensitive teeth after scaling and root planing were included in this study and randomly divided into two groups each containing 15 patients:

Group I received Pro-Argin technology (8\% arginine and calcium carbonate)

Group II received Novamin technology (calcium sodium phosphosilicate, a bioactive glass) containing desensitizing toothpaste. There were no dropouts in the present study.

The mean SCASS score for Novamin at baseline and posttreatment was 2.40 and 0.13 respectively. The mean difference between the two groups was statistically highly significant $(\mathrm{p}<0.001)$ (Table 1 and Graph 1).

The mean SCASS score for Pro-Argin at baseline and posttreatment was 2.33 and 0.07 respectively. The mean difference between the two groups was statistically highly significant $(\mathrm{p}<0.001)$ (Table 2 and Graph 1).

Table 1: Statistical analysis for SCASS and VAS scores of Novamin at baseline and 1 week posttreatment

\begin{tabular}{llllllcc}
\hline Parameter & Time interval & Mean & Std. dev & Std. error of mean & Mean difference & z-value & $p$-value \\
\hline Schiff cold air & Baseline & 2.40 & 0.51 & 0.13 & 2.267 & -3.508 & $<0.001^{*}$ \\
sensitivity scale & After 1 week & 0.13 & 0.35 & 0.09 & & \\
Visual analog & Baseline & 6.67 & 2.44 & 0.63 & & -3.507 & $<0.001^{*}$ \\
scale & After 1 week & 0.20 & 0.56 & 0.14 & & \\
\hline
\end{tabular}

The reduction in mean SCASC score from baseline to 1 week was found to be statistically significant $(P<0.001)$

Table 2: Statistical analysis for SCASS and VAS scores of Pro-Argin at baseline and 1 week posttreatment

\begin{tabular}{|c|c|c|c|c|c|c|c|}
\hline Parameter & Time interval & Mean & Std. dev & Std. error of mean & Mean difference & z-value & $p$-value \\
\hline Schiff cold air & Baseline & 2.33 & 0.49 & 0.13 & 2.267 & -3.578 & $<0.001^{*}$ \\
\hline sensitivity scale & After 1 week & 0.07 & 0.26 & 0.07 & & & \\
\hline Visual analog & Baseline & 6.33 & 2.29 & 0.59 & 6.200 & -3.573 & $<0.001^{*}$ \\
\hline scale & After 1 week & 0.13 & 0.52 & 0.13 & & & \\
\hline
\end{tabular}

The reduction in mean VAS score from baseline to 1 week was found to be statistically significant $(P<0.001)$ 
Table 3: Statistical analysis for SCASS between Novamin and Pro-Argin at baseline and 1 week posttreatment

\begin{tabular}{lllllllc}
\hline Schiff cold air sensitivity scale & Technology & Mean & Std. dev & Std. error of mean & Mean difference & z-value & $p$-value \\
\hline Baseline & Novamin & 2.40 & 0.51 & 0.13 & 0.067 & -0.372 & 0.710 \\
& Pro-Argine & 2.33 & 0.49 & 0.13 & & 0.067 & -0.598 \\
After 1 week & Novamin & 0.13 & 0.35 & 0.09 & 0.550 \\
& Pro-Argine & 0.07 & 0.26 & 0.07 & & \\
\hline
\end{tabular}

Table 4: Statistical analysis for VAS between Novamin and Pro-Argin at baseline and 1 week posttreatment

\begin{tabular}{llllllll}
\hline Visual analog scale & Technology & Mean & Std. dev & Std. error of mean & Mean difference & z-value & $p$-value \\
\hline Baseline & Novamin & 6.67 & 2.44 & 0.63 & 0.333 & -0.392 & 0.695 \\
& Pro-Argine & 6.33 & 2.29 & 0.59 & & 0.067 & -0.558 \\
After 1 week & Novamin & 0.20 & 0.56 & 0.14 & 0.577 \\
& Pro-Argine & 0.13 & 0.52 & 0.13 & & & \\
\hline
\end{tabular}

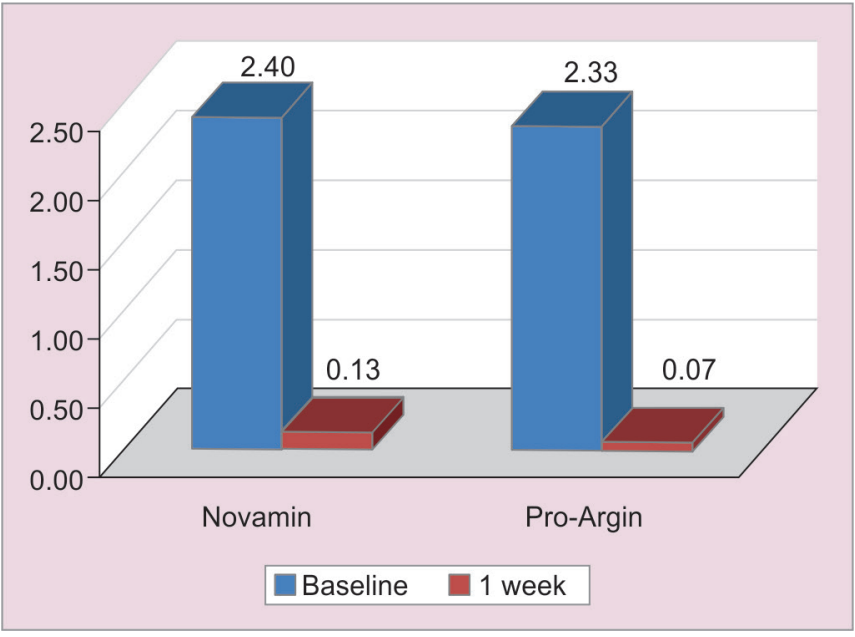

Graph 1: Mean SCASS score recorded in the two technologies

The mean SCASS scores at baseline for Novamin and Pro-Argin were 2.40 and 2.33 respectively. The difference between the two groups was not statistically significant (Table 3 and Graph 1).

The mean SCASS scores for Novamin and Pro-Argin posttreatment were 0.13 and 0.07 respectively. The difference between the two groups was not statistically significant (Table 3 and Graph 1) ( $\mathrm{p}>0.05)$.

The difference in mean SCASS score between baseline and posttreatment for Novamin and Pro-Argin was not statistically significant.

The mean VAS score for Novamin at baseline and posttreatment was 6.67 and 0.20 respectively. The mean difference between the two groups was statistically highly significant $(\mathrm{p}<0.001)$ (Table 1 and Graph 2).

The mean VAS score for Pro-Argin at baseline and posttreatment was 6.33 and 0.13 respectively. The mean difference between the two groups was statistically highly significant $(\mathrm{p}<0.001)$ (Table 2 and Graph 2).

The mean VAS scores at baseline for Novamin and Pro-Argin were 6.67 and 6.33 respectively. The difference between the two groups was not statistically significant (Table 4 and Graph 2) ( $p>0.05)$.

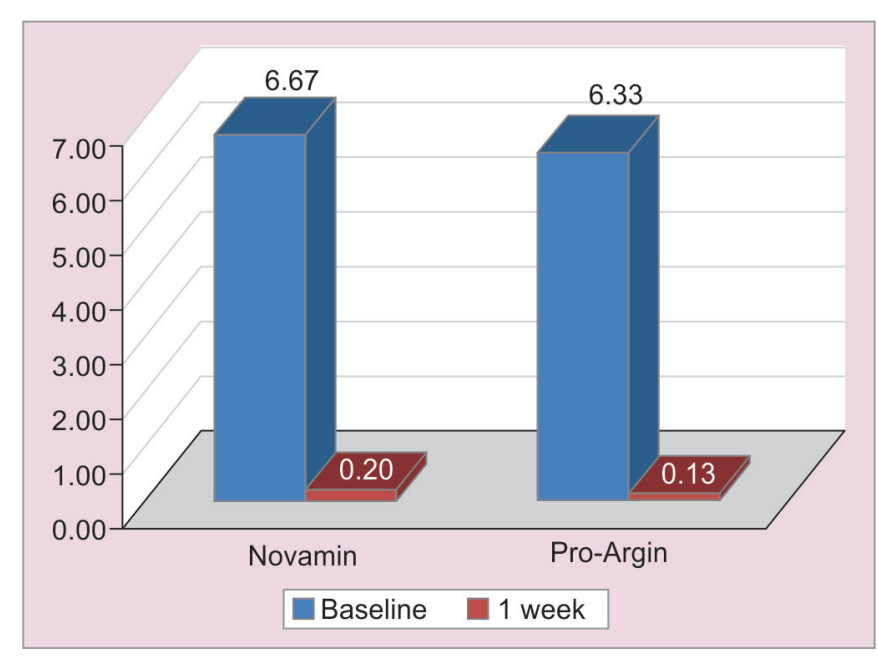

Graph 2: Mean VAS score recorded in the two technologies

The mean VAS scores for Novamin and Pro-Argin posttreatment were 0.20 and 0.13 respectively. The difference between the two groups was not statistically significant (Table 4 and Graph 2) ( $p>0.05)$.

The difference in mean VAS score between baseline and posttreatment for Novamin and Pro-Argin was not statistically significant.

At 2 nd and 4th weeks, the scores were constant for both the groups (score -0 ).

Using Mann-Whitney test, we found no statistically significant differences in the score between the two desensitizing toothpastes. But, there was a statistically significant difference between the baseline and followup scores done consecutively for 4 weeks for individual toothpastes.

\section{DISCUSSION}

The $\mathrm{DH}$ is a problem that plagues many patients. The periodontal procedures used to remove plaque and associated damaged tissue are known to increase sensitivity in a transient manner. Products and techniques used for treatment of $\mathrm{DH}$ are diverse, suggesting uncertainty 
among dentists about the best way to treat patients, as well as dissatisfaction with outcomes of available treatments. The development of a therapy that can provide both immediate relief following professional application and a lasting desensitizing effect for a significant time period after use would be of great assistance to clinicians in dealing with $\mathrm{DH}^{17,18}$

The results of the present study demonstrate comparable clinical effectiveness for the two dentifrices with significant reductions in measures of tooth sensitivity, observed across all measures, at all predefined time points, i.e., groups I and II respectively, vs baseline. This is in agreement with the studies by Salian et $\mathrm{al}^{19}{ }^{19}$ Sharma et $\mathrm{al}^{20}{ }^{20}$ West et $\mathrm{al}^{21}$ and Litkowski and Greenspan. ${ }^{22}$

Using dentifrice as a delivery vehicle for Novamin and Pro-Argin is likely to be economical and is not technique sensitive. Therefore, it could offer an excellent at-home treatment.

A comparative study by Parkinson and Willson ${ }^{24}$ in 2011 concluded that calcium sodium phosphosilicate (Novamin) imparts significant level of dentinal occlusion with durable occlusive deposits following four days of twice daily brushing in vitro.

In early studies done by Kleinberg and Sensistat ${ }^{12}$ in 2002 demonstrated that application of the arginine calcium carbonate in office desensitizing paste to teeth exhibiting sensitivity following dental prophylaxis resulted in instant relief from discomfort and that relief lasted for 28 days after a single application.

Large and clinically relevant improvement from baseline to 1 week was observed for the two treatment groups using air blast method and cold sensitivity test wherein at 2 nd and 4th week, no sensitivity was observed among all the 30 patients depicting a score of 0 .

No significant differences between treatment groups were apparent at any of the time points. Given the comprehensive improvements in sensitivity relief in both treatment groups across all the efficacy measures, these results suggest a comparable level of performance between the test products with no apparent difference between the Novamin and Pro-Argin formulations.

This could possibly be due to the fact that both the active agents have been supplied using dentifrice as a delivery vehicle and the excipient (nonactive) agents in the dentifrice may serve to occlude dentinal tubules over time.

Also, this effect could be related to a natural decrease in $\mathrm{DH}$ over time, or because of patient perception of a decrease in symptoms by virtue of participation in a clinical trial, or may be due to placebo products actually providing some degree of relief from DH.

The results of the present study may have to be extrapolated with caution given the small sample size and lack of accounting for the placebo effect and the Haw thorne effect. However, longitudinal studies involving larger sample size with longer follow-up period will be required to confirm the effect of these two formulations on a long-term basis.

\section{CONCLUSION}

Therefore, within the limitations of the study, we found no statistically significant difference in the efficacy of the two desensitizing toothpastes.

\section{REFERENCES}

1. Canadian Advisory Board on Dentin Hypersensitivity. Consensus-based recommendations for the diagnosis and management of dentin hypersensitivity. J Can Dent Assoc 2003 Apr;69(4):221-226.

2. Holland GR, Narhi MN, Addy M, Gangarosa L, Orchardson R. Guidelines for the design and conduct of clinical trials on dentine hypersensitivity. J Clin Periodontol 1997 Nov;24(11): 808-813.

3. Gibson B, Boiko OV, Baker S, Robinson PG, Barlow A, Player T, Locker D. The everyday impact of dentine sensitivity: personal and functional aspects. Soc Sci Dent 2010;1(1):11-20.

4. Barlow AP, He J, Tian C, Jeffery P, Mason SC, Tai BJ, Jiang H, Rees GD, Du MQ. A comparative evaluation of the efficacy of two novel desensitising dentifrices. Int J Dent 2012;2012:896143.

5. Addy M. Etiology and clinical implications of dentine hypersensitivity. Dent Clin North Am 1990 Jul;34(3):503-514.

6. Orchardson R, Collins WJ. Clinical features of hypersensitive teeth. Br Dent J 1987 Apr;162(7):253-256.

7. Bamise CT, Olusile AO, Oginni AO. An analysis of the etiological and predisposing factors related to dentin hypersensitivity. J Contemp Dent Pract 2008 Jul;9(5):52-59.

8. van Steenberghe D, Garmyn P, Geers L, Hendrickx E, Maréchal M, Huizar K, Kristofferson A, Meyer-Rosberg K, Vandenhoven G. Patients' experience of pain and discomfort during instrumentation in the diagnosis and non-surgical treatment of periodontitis. J Periodontol 2004 Nov;75(11): 1465-1470.

9. Cunha-Cruz J, Wataha JC, Zhou L, Manning W, Trantow M, Bettendorf MM, Heaton LJ, Berg J. Treating dentin hypersensitivity: therapeutic choices made by dentists of the northwest PRECEDENT network. J Am Dent Assoc 2010 Sep;141(9):1097-1105.

10. Bartold PM. Dentinal hypersensitivity: a review. Aust Dent J 2006 Sep;51(3):212-218.

11. Panagakos F, Schiff T, Guignon A. Dentin hypersensitivity: effective treatment with an in-office desensitizing paste containing $8 \%$ arginine and calcium carbonate. Am J Dent 2009 Mar;22 Spec No A:3A-7A.

12. Kleinberg I. Sensistat. A new saliva-based composition for simple and effective treatment of dentinal sensitivity pain. Dent Today 2002 Dec;21(12):42-47.

13. Jennings DT, McKenzie KM, Greenspan DC, Clark A, Clark AE. Quantitative analysis of tubule occlusion using NovaMin ${ }^{\circledR}$ (sodium calcium phosphosilicate). J Dent Res 2004;83 Spec Issue A:2416.

14. Litkowski, LJ.; Hack, GD.; Sheaffer, HB.; Greenspan, DC. Occlusion of dentintubules by Bioglass ${ }^{\circledR}$. In: Bioceramics 10, 
Proceedings of the 10th International Symposium on Ceramics in Medicine, Paris, France; 1997. p. 10.

15. Zhong JP, Greenspan DC, Feng JW. A microstructural examination of apatite induced by Bioglass in vitro. J Mater Sci Mater Med 2002 Mar;13(3):321-326.

16. Schiff T, Dotson M, Cohen S, De Vizio W, McCool J, Volpe A. Efficacy of a dentifrice containing potassium nitrate, soluble pyrophosphate, PVM/MA copolymer, and sodium fluoride on dentinal hypersensitivity: a twelve-week clinical study. J Clin Dent 1994;5 Spec No:87-92.

17. Schiff T, Delgado E, Zhang YP, Cummins D, DeVizio W, Mateo LR. Clinical evaluation of the efficacy of an in-office desensitizing paste containing $8 \%$ arginine and calcium carbonate in providing instant and lasting relief of dentin hypersensitivity. Am J Dent 2009 Mar;22 Spec No A: 8A-15A.

18. Kapferer I, Pflug C, Kisielewsky I, Giesinger J, Beier US, Dumfahrt H. Instant dentin hypersensitivity relief of a single topical application of an in-office desensitizing paste containing $8 \%$ arginine and calcium carbonate: a split-mouth, randomized-controlled study. Acta Odontol Scand 2013 May-Jul;71(3-4):994-999.
19. Salian S, Thakur S, Kulkarni S, LaTorre G. A randomized controlled clinical study evaluating the efficacy of two desensitizing dentifrices. J Clin Dent 2010;21(3):82-87.

20. Sharma N, Roy S, Kakar A, Greenspan DC, Scott R. A clinical study comparing oral formulations containing $7.5 \%$ calcium sodium phosphosilicate (NovaMin), 5\% potassium nitrate, and $0.4 \%$ stannous fluoride for the management of dentin hypersensitivity. J Clin Dent 2010;21(3):88-92.

21. West NX, Macdonald EL, Jones SB, Claydon NC, Hughes N, Jeffery P. Randomized in situ clinical study comparing the ability of two new desensitizing toothpaste technologies to occlude patent dentin tubules. J Clin Dent 2011;22(3):82-89.

22. Litkowski L, Greenspan DC. A clinical study of the effect of calcium sodium phosphosilicate on dentin hypersensitivity proof of principle. J Clin Dent 2010;21(3):77-81.

23. Acharya AB, Surve SM, Thakur SL. A clinical study of the effect of calcium sodium phosphosilicate on dentin hypersensitivity. J Clin Exp Dent 2013 Feb;5(1):e18-e22.

24. Parkinson CR, Willson RJ. A comparative in vitro study investigating the occlusion and mineralization properties of commercial toothpastes in a four-day dentin disc model. J Clin Dent 2011;22(3):74-81. 